\title{
Whole-cell Cryo-ET Structural Studies of Respiratory Syncytial Virus
}

\author{
Bryan Sibert, Jae Yang, Joseph Kim, Juleen Dickson and Elizabeth Wright \\ University of Wisconsin-Madison, Madison, Wisconsin, United States
}

Respiratory syncytial virus (RSV) is the leading cause of bronchitis in infants and a significant cause of respiratory illness in adults [1]. Though a prophylactic treatment with monoclonal antibodies against the fusion protein $(\mathrm{F})$ is protective in at-risk infants, there is currently no approved cure or vaccine for RSV. RSV is a member of the Paramyxoviridae family including measles virus, parainfluenza virus, and other clinically relevant negative-strand RNA viruses. RSV is an enveloped filamentous virus that ranges in length from $\sim 1 \mu \mathrm{m}$ to $>10 \mu \mathrm{m}$ and varies in diameter with an average of $\sim 130 \mathrm{~nm}$. The filaments are studded with the viral glycoproteins, predominantly F [2]. RSV matrix (M) is localized immediately interior to, and peripherally associated with, the viral membrane $[2,3]$. Though there are several other structural proteins, expression of $\mathrm{M}$ and $\mathrm{F}$ along with viral phosphoprotein $(\mathrm{P})$ is sufficient for formation of filamentous virus-like particles demonstrating the important role of $\mathrm{M}$ and $\mathrm{F}$ in determining virus structure [4].

Visualizing the organization of RSV $\mathrm{M}$ is challenging for a number of reasons including its small size $(\sim 28 \mathrm{kD})$, association with the membrane, and sensitivity to mechanical stress during purification [5]. To overcome these challenges, we used whole cell cryo-electron tomography (cryo-ET) to image RSV released from human airway epithelial cells that have been cultured directly on EM grids. Using this technique, we are able to resolve a lattice-like density in the expected location of RSV M (Figure 1A-C). The lattice is present throughout the majority of the virion, though irregular or incomplete in places, including gaps in regions of high curvature. Work is ongoing to further characterize this lattice. Preliminary analysis indicates an approximately $5.2 \mathrm{~nm}$ spacing of the lattice consistent with the spacing of $M$ in related viruses and the unit cell size of a crystallized RSV M dimer $[6,7,8]$.

To further investigate the structure and function of this $\mathrm{M}$ lattice, we examined virions from recombinant RSV containing a mutation of M amino acid threonine 205 to alanine (MT205A), previously shown to impact viral fitness and thought to regulate high-order M oligomerization [9]. As in wild-type, the matrix lattice was present in these mutant virions with the same $\sim 5.2 \mathrm{~nm}$ spacing. However, relative to wild-type, we observed a greater number of irregular virions with an extension of the M layer into the virion, with no apparent association with viral membrane. Additionally, several virions appeared to be only sparsely covered with glycoproteins. These data suggest that M-T205 is important for M-lipid and M-F interactions. Analysis of additional mutants, including mutations at the $\mathrm{M}$ dimer interface, is underway to further characterize the function of essential $M$ interactions. A better understanding of the determinants of RSV viral structure will aid in the development of attenuated vaccine strains and therapeutics [10]. 

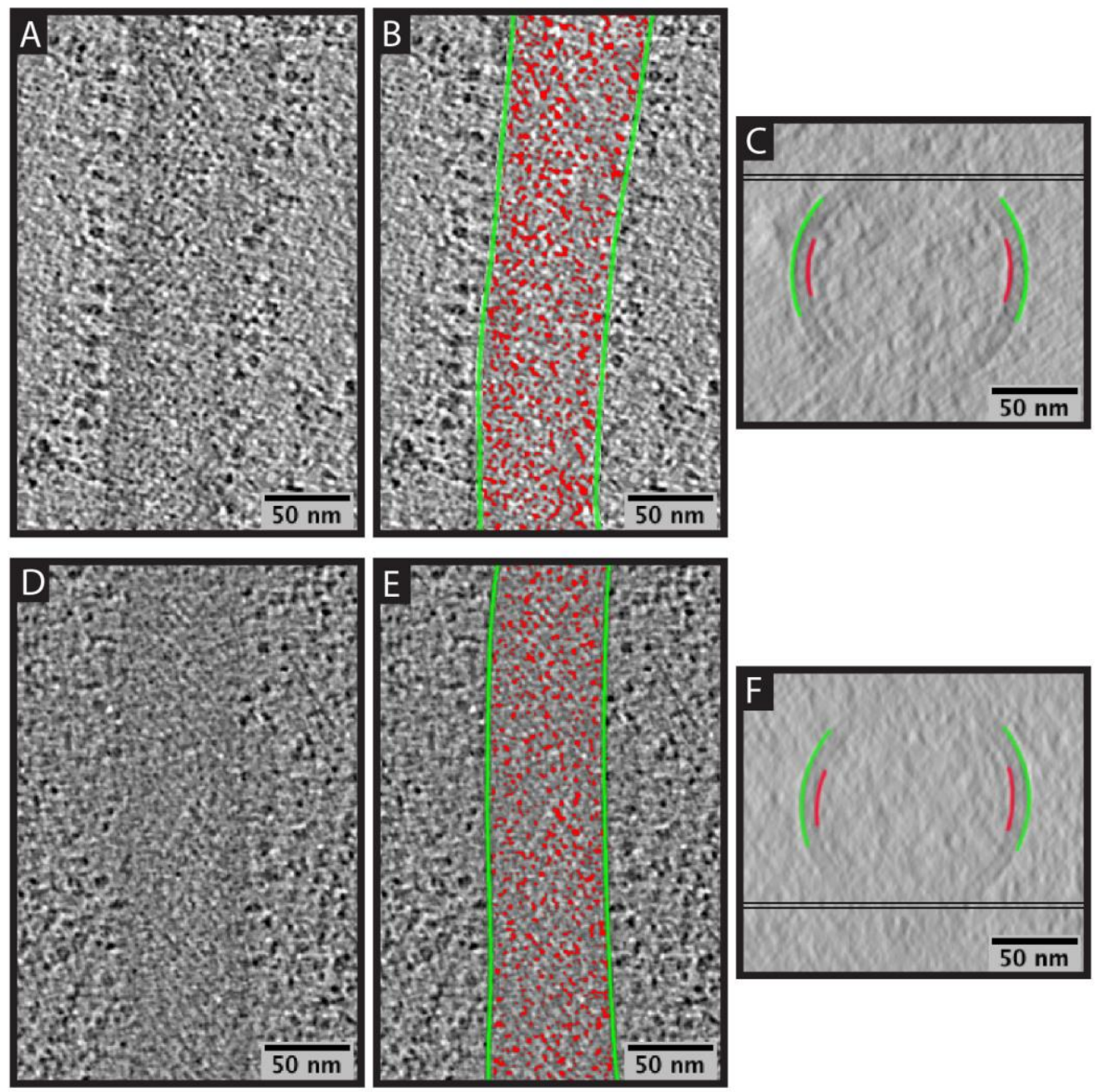

Figure 1. Cryo-ET of RSV reveals the lattice-like density of the M protein layer. A) Average Z-projection of three X,Y slices (2.7 nm thick) from a tomogram of RSV. B) Image from A with densities above threshold and within the virus shown in red to better visualize the lattice-like organization of the $M$ layer. The viral membrane is segmented in green. (C) Average Y-projection of ten X,Z slices (8.9 nm thick) from the region in $\mathrm{A}$. The viral membrane and $\mathrm{M}$ layer are partially segmented in green and red respectively. The Z-layer projected in A \& B is indicated by black bars. (D,E,F) As in (A,B,C) with an RSV virion containing the MT205A mutation.

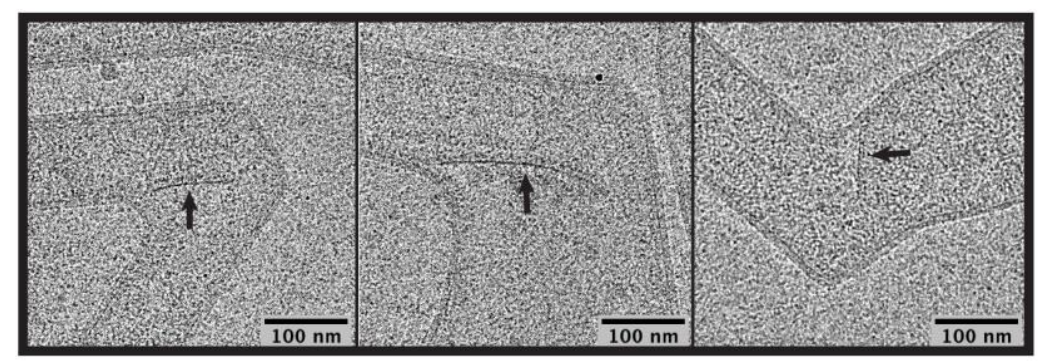

Figure 2. Aberrant M-like densities can be seen in cryo-EM micrographs of RSV virions with the MT205A mutation. 


\section{References}

[1] Walsh EE. Clin Chest Med. 38(1) (2017), p. 29.

[2] Ke Z., et al. Viruses 10(8) (2018), p. 446.

[3] Liljerooos L., et al. PNAS 110(27) (2013), p. 11133.

[4] Meshram CD., et al. J Virol. 90(23) (2016), p. 10612.

[5] Kiss G., et al. J Virol. 88(13) (2014), p. 7602.

[6] Förster A., et al. J Virol. 89(8) (2015), p. 4624.

[7] Ke Z., et al. Nat Commun. 9(1) (2018), p. 1736.

[8] Battisti AJ., et al. PNAS 109(35) (2012), p. 13996.

[9] Bajorek M., et al. J Virol. 88(11) (2014), p. 6380.

[10] This research was supported in part by funds from the University of Wisconsin, Madison and the National Institutes of Health (R01GM114561 and R01GM104540-03S1) to E.R.W. The authors gratefully acknowledge use of facilities and instrumentation at the UW-Madison Wisconsin Centers for Nanoscale Technology (wcnt.wisc.edu) partially supported by the NSF through the University of Wisconsin Materials Research Science and Engineering Center (DMR-1720415). A portion of this research was supported by NIH grant U24GM129547 and performed at the PNCC at OHSU and accessed through EMSL (grid.436923.9), a DOE Office of Science User Facility sponsored by the Office of Biological and Environmental Research. 